\title{
Assessing colposcopic accuracy for high- grade squamous intraepithelial lesion detection: a retrospective, cohort study
}

Anying Bai ${ }^{1 \dagger}$, Jiaxu Wang ${ }^{1 \dagger}$, Qing $\mathrm{Li}^{2}$, Samuel Seery ${ }^{3}$, Peng Xue ${ }^{1 *}$ and Yu Jiang ${ }^{1 *}$

\begin{abstract}
Background: Inappropriate management of high-grade squamous intraepithelial lesions (HSIL) may be the result of an inaccurate colposcopic diagnosis. The aim of this study was to assess colposcopic performance in identifying $\mathrm{HSIL}+$ cases and to analyze the associated clinical factors.

Methods: Records from 1130 patients admitted to Shenzhen Maternal and Child Healthcare Hospital from 12th January, 2018 up until 30th December, 2018 were retrospectively collected, and included demographics, cytological results, HPV status, transformation zone type, number of cervical biopsy sites, colposcopists' competencies, colposcopic impressions, as well as histopathological results. Colposcopy was carried out using 2011 colposcopic terminology from the International Federation of Cervical Pathology and Colposcopy. Logistic regression modelling was implemented for uni- and multivariate analyses. A forward stepwise approach was adopted in order to identify variables associated with colposcopic accuracy. Histopathologic results were taken as the comparative gold standard.
\end{abstract}

Results: Data from 1130 patient records were collated and analyzed. Colposcopy was 69.7\% accurate in identifying $\mathrm{HSIL}+$ cases. Positive predictive value, negative predictive value, sensitivity and specificity of detecting HSIL or more $(\mathrm{HSIL+})$ were $35.53 \%, 64.47 \%, 42.35 \%$ and $77.60 \%$, respectively. Multivariate analysis highlighted the number of biopsies, cytology, and transformation zone type as independent factors. Age and HPV subtype did not appear to statistically correlate with high-grade lesion/carcinoma.

Conclusion: Evidence presented here suggests that colposcopy is only $69.7 \%$ accurate at diagnosing HSIL. Even though not all HSIL will progress into cancer it is considered pre-cancerous and therefore early identification will save lives. The number of biopsies, cytology and transformation zone type appear to be predictors of misdiagnosis and therefore should be considered during clinical consultations and by way of further research.

Keywords: Cervical cancer, Colposcopy, Cervical biopsy, High-grade squamous intraepithelial lesion

*Correspondence: xuepeng_pumc@foxmail.com; jiangyu@pumc.edu.cn ${ }^{\dagger}$ Anying Bai and Jiaxu Wang have contributed equally to this work and share first authorship

${ }^{1}$ Department of Epidemiology and Biostatistics, School of Population Medicine and Public Health, Chinese Academy of Medical Sciences and Peking Union Medical College, Beijing 100730, China

Full list of author information is available at the end of the article

\section{Background}

Cervical cancer is one of the most prevalent genital tumors and poses a threat to every women's health [1]. In 2020, cervical cancer caused approximately 340,000 deaths with a further 600,000 new cases recorded. This accounts $3.4 \%$ of all deaths and $3.3 \%$ of all cancers incidents, globally. In China alone, cervical cancer is of growing concern [2], where according to national cancer statistics, the incidence of cervical cancer in 2015 was 
9.89 per 10,000 with a 3.05 per 10,000 mortality rate [3]. Human papillomavirus (HPV) infection is universally recognized as a causative agent in the development of cervical intraepithelial neoplasia (CIN) and squamous intraepithelial lesions. These can be benign but are considered pre-cancerous and often develop into invasive cervical carcinoma [4]. As precursors to invasive squamous carcinoma, over one third of all high-grade squamous intraepithelial lesions (HSIL) and CIN grades II and III, progress into cervical cancer over a period of between 10 and 15 years [5].

In order to combat the global prevalence and mortality of cervical cancer most countries have implemented screening programs which utilize cervical cytology and/ or HPV testing, and then colposcopy when screening finds abnormal cells in the cervix. Therefore, colposcopy is an indispensable tool for early detection with accurate use benefiting women by reducing the number of unnecessary biopsies, conization procedures, as well as the frequency of cauterization therapy for cervical erosions [6]. This means, there is a great deal of avoidable stress caused by diagnostic inaccuracies and discrepancies between colposcopic and pathologic diagnosis are known [7]. Colposcopy is considered a subjective procedure, which is dependent upon a clinician's assessment. Many factors can therefore bias diagnosis, such as knowing cytologic results, or HPV subtypes and transformation zone types. Therefore, it remains necessary to identify reasons for diagnostic inaccuracies, in order to reduce unnecessary stress caused and improve outcomes.

In 2011, the International Federation of Cervical Pathology and Colposcopy (IFCPC) provided a new terminology system based on versions developed in 1975 [8], 1990 [9]. and 2002 [10]. This new system provides a more comprehensive understanding with evidence-based reclassifications of some abnormal colposcopic findings [6]. However, there are few studies which evaluate the 2011 IFCPC terminology and despite the prevalence of cervical cancer in China there are very few which utilize the IFCPC system across a Chinese sample. Therefore, it is not only important to identify and assess potential causes of colposcopic inaccuracies but also to understand diversity and variance. The primary aim of this study was to assess levels of agreement between colposcopy and cervical biopsy in identifying HSIL. However, this also necessitates an investigation into the associated clinicopathological factors affecting diagnostic accuracy.

\section{Methods}

\section{Study population}

Patient records for those who underwent colposcopic examination at Shenzhen Maternal and Child Health Hospital from 12th January 2018 to 30th December 2018 were retrospectively analyzed. Patients without adequate colposcopy impressions, HPV, cytology or histopathological results, and those who did not have basic demographic information, were excluded. The final sample consisted of 1130 patients.

Demographics and clinical characteristics including age, cytological examination results, HPV status, transformation zone type, number of cervical biopsy sites, colposcopy results and pathological results, were collected. This study was conducted ethically in accordance with the World Medical Association Declaration of Helsinki. This study was approved by the institutional review board (IRB) of Shenzhen maternal and Child Health Hospital (No. 164), and the need for informed consent was waived by the IRB of SZMCHH due to the retrospective nature of archived datasets and fully anonymized personal information.

\section{HPV and cytology subtypes/categories}

HPV genotyping was detected in liquid-based cytology specimens collected using the HPV GenoArray test kit (HybriBio Ltd). This kit is capable of identifying 15 highrisk HPV (hrHPV) types $(16,18,31,33,35,39,45,51,52$, $56,58,59,65,66$, and 68 ) [11]. In this study, patients were categorized as HPV negative, HPV 16/18 and other HRHPV positive type.

Thinprep cytologic test (TCT, Hologic, USA) were used to perform cytologic analysis. Results were reported according to the Bethesda System [12] and categorized into five classes: negative for intraepithelial lesion or malignancy (NILM), atypical squamous cells of undetermined significance (ASCUS), atypical squamous cells-cannot exclude high-grade squamous intraepithelial lesion (ASC-H), low-grade squamous intraepithelial lesion (LSIL), and HSIL and/or squamous cell carcinoma (HSIL/SCC).

\section{Colposcopy and biopsy}

General assessment was conducted in accordance with the 2011 IFCPC colposcopic terminology for the cervix [13] which includes transformation zone types 1, 2 or 3 . All colposcopies were performed by gynecologists using an electronic colposcope (Goldway Ltd). The number of biopsies was reported with histopathologies, and pathologic results were taken as the gold standard.

Patients with confirmatory colposcopic and pathologic results for HSIL+ or $<$ HSIL were categorised into the 'concordant' group. All others were assigned to the group labelled 'discordant'. The accordance rate is the percentage of patients with confirmatory colposcopic and histopathologic findings. Overdiagnosis was considered present when histopathologic results highlighted less advanced lesions than colposcopic examination. 
Underdiagnosis was considered to have occurred when histopathological results highlighted more advanced lesion/s than colposcopic examination.

\section{Statistical analysis}

The characteristics of the concordant and disconcordant groups were compared using Pearson's $\chi^{2}$ test for qualitative variables and Student's t-test for quantitative variables. Differences between underdiagnosis, overdiagnosis and accordance between subgroups of all the patients were examined using $\chi^{2}$ tests or Fisher's exact tests.

Logistic regression modelling was used for uni- and multivariate analyses. A forward stepwise approach was implemented to identify variables influencing colposcopic accuracy.

All $p$ values are two-sided, with the threshold for statistical significance set at 0.05 . All statistical analyses were performed using SAS software (version 9.4). All methods were performed in accordance with the Declaration of Helsinki.

Table 1 Characteristics of all patients for subgroup analysis

\begin{tabular}{llll}
\hline Variables & $\begin{array}{l}\text { Discordant } \\
\mathbf{n = 3 4 3}\end{array}$ & $\begin{array}{l}\text { Concordant } \\
\mathbf{n = 7 8 7}\end{array}$ & $\boldsymbol{p}$ \\
\hline Age categories & & & 0.031 \\
$<30$ & $37.74 \%(n=80)$ & $62.26 \%(n=132)$ & \\
$30-45$ & $28.36 \%(n=226)$ & $71.64 \%(n=571)$ & \\
$>45$ & $30.58 \%(n=37)$ & $69.42 \%(n=84)$ & \\
Cytology results & & & $<0.001$ \\
NILM & $24.34 \%(n=176)$ & $75.66 \%(n=547)$ & \\
ASC-US & $42.44 \%(n=101)$ & $57.56 \%(n=137)$ & \\
LSIL & $39.58 \%(n=57)$ & $60.42 \%(n=87)$ & \\
ASC-H & $66.67 \%(n=4)$ & $33.33 \%(n=2)$ & \\
HSIL & $26.32 \% n=5)$ & $73.68 \%(n=14)$ & \\
HPV subtypes & & & \\
Negative & $30.59 \%(n=26)$ & $69.41 \%(n=59)$ & \\
Other HR-HPV positive & $37.91 \%(n=127)$ & $62.09 \%(n=208)$ & \\
HPV16/18 & $26.76 \%(n=190)$ & $73.24 \%(n=520)$ & \\
Transformation zone type & & & \\
TZ 1 & $20.20 \%(n=61)$ & $79.80 \%(n=241)$ & \\
TZ2 & $32.56 \%(n=42)$ & $67.44 \%(n=87)$ & \\
TZ3 & $34.33 \%(n=240)$ & $65.67 \%(n=459)$ & \\
Number of biopsies & $1.1603 \pm 2.1180$ & $1.4727 \pm 1.5920$ & 0.006 \\
\hline
\end{tabular}

\section{Results}

Clinical characteristics of study population

Table 1 provides summaries of clinical characteristics for all patients and the results of subgroup analysis including age of the women at the time of examination, cytologic results, HPV genotype, and transformation zone information. Of the 1130 patients included in this study, $69.64 \%(n=787)$ received a consistent diagnosis, whereas $30.35 \%(n=343)$ of all HSIL + patients were not identified through colposcopy. The diagnostic accuracy of HSIL+ cases correlated positively with increasing age. That is, we observed $62.26 \%$ accuracy for those below 30 years, $71.64 \%$ for those aged between 30 and 45 , and $69.42 \%$ for age 45 or older $(p=0.031)$.

Only $73.7 \%$ of all HSIL cases were detected through colposcopy. Cytologic analysis found consistently benign results across subgroups, as follows: NILM (75.66\%), ASC-US (57.56\%), LSIL (60.42\%), and ASC-H (33.33\%). When detecting HSIL+ cases there was a significant difference across transformation zones $(p<0.001)$ with TZ 1 associated with the highest diagnostic accuracy $(79.80 \%)$, TZ 2 (67.44\%) and TZ 3 (65.67\%).

The consistent rate in patients with HPV 16 or 18 genotypes was $73.24 \%$, which was higher than other HPV positive genotypes, but marginally lower among patients who tested negative for $\operatorname{HPV}(p=0.001)$.

Tables 2, 3, 4 and 5 provide results of subgroup analysis i.e. age of the women at the time of examination, cytology results, HPV subtypes and transformation zone types. The accuracy for HSIL+ detection decreased with increasing age, and HSIL+cases were more likely to be missed among older women (please see Table 2. for details). At the same time, the chances of underdiagnosing and overdiagnosing decreased among HSIL patients more than patients with other subtypes $(p<0.001)$. See Table 3 for further comparisons.

The accordance rate between colposcopic and pathologic examinations was highest among HPV 16/18 cases. While the rate of overdiagnosis was worse in negative patients, the rate of underdiagnosis was highest among patients with other HR-HPV positive results (Table 4). Differences in detection accuracy among HSIL+ patients with TZ 1, TZ 2, or TZ 3 were significant $(p<0.001)$. The rate of overdiagnosis was worse in women with $\mathrm{TZ} 3$ (19.74\%), whereas women with TZ 1 or TZ 2 appear to

Table 2 Accordance rate, over- and underdiagnosis of HSIL+ relative to patients' age ( $p=0.190)$

\begin{tabular}{lllll}
\hline & $\mathbf{0 - 3 0}$ years $(\mathbf{n}=\mathbf{2 6 8})$ & $\mathbf{3 1 - 4 5}$ years $(\mathbf{n}=\mathbf{7 4 1 )}$ & > $\mathbf{4 5}$ years $(\mathbf{n}=\mathbf{1 3 9 )}$ & Total \\
\hline Overdiagnosis & $19.78 \%(n=53)$ & $17.14 \%(n=127)$ & $11.51 \%(n=16)$ & 196 \\
Accordance & $65.67 \% n=176)$ & $71.12 \%(n=527)$ & $60.43 \%(n=84)$ & 787 \\
Underdiagnosis & $14.55 \%(n=39)$ & $11.74 \%(n=87)$ & $15.11 \%(n=21)$ & 147 \\
\hline
\end{tabular}


Table 3 Accordance rate, over- and underdiagnosis of HSIL+ relative to cytologic results $(p<0.001)$

\begin{tabular}{lccccc}
\hline & NILM $(\mathbf{n}=\mathbf{7 2 3})$ & ASC-US $(\mathbf{n}=\mathbf{2 3 8})$ & LSIL $(\mathbf{n}=\mathbf{1 4 4})$ & ASC-H $(\mathbf{n}=\mathbf{6})$ & HSIL (N= 19) \\
\hline Overdiagnosis & $21.72 \%(n=157)$ & $9.66 \%(n=23)$ & $8.33 \%(n=12)$ & $33.33 \%(n=2)$ & $10.53 \%(n=2)$ \\
Accordance & $75.66 \%(n=547)$ & $57.56 \%(n=137)$ & $60.42 \%(n=87)$ & $33.33 \%(n=2)$ & $73.68 \%(n=14)$ \\
Underdiagnosis & $2.63 \%(n=19)$ & $32.77 \%(n=78)$ & $31.25 \%(n=45)$ & $33.33 \%(n=2)$ & $15.79 \%(n=3)$ \\
\hline
\end{tabular}

Table 4 Accordance rate, over- and underdiagnosis of $\mathrm{HSIL}+$ relative to HPV subtypes $(p<0.001)$

\begin{tabular}{llll}
\hline & Negative & HPV16/18 & $\begin{array}{l}\text { Other HR-HPV } \\
\text { positive }\end{array}$ \\
\hline Overdiagnosis & $24.71 \%(n=21)$ & $13.66 \%(n=97)$ & $23.28 \%(n=78)$ \\
Accordance & $69.41 \%(n=59)$ & $73.42 \%(n=520)$ & $62.09 \%(n=208)$ \\
Underdiagnosis & $5.88 \%(n=5))$ & $13.10 \%(n=93)$ & $14.63 \%(n=49)$ \\
\hline
\end{tabular}

Table 5 Accordance rate, over- and underdiagnosis of $\mathrm{HSIL}+$ relative to transformation zone type $(p<0.001)$

\begin{tabular}{lllll}
\hline & TZ 1 $(\mathbf{N}=\mathbf{3 0 2})$ & $\begin{array}{l}\text { TZ 2 } \\
(\mathbf{N}=129)\end{array}$ & TZ 3 $(\mathbf{N}=\mathbf{6 9 9})$ & Total \\
\hline Overdiagnosis & $\begin{array}{l}12.91 \% \\
(n=39)\end{array}$ & $14.73 \%(n=19)$ & $\begin{array}{l}19.74 \% \\
(n=138)\end{array}$ & 196 \\
Accordance & $\begin{array}{l}79.88 \% \\
(n=241)\end{array}$ & $67.44 \%(n=87)$ & $\begin{array}{l}65.67 \% \\
(n=459)\end{array}$ & 787 \\
$\begin{array}{l}\text { Underdiag- } \\
\text { nosis }\end{array}$ & $7.28 \%(n=22)$ & $17.83 \%(n=23)$ & $\begin{array}{l}14.59 \% \\
(n=102)\end{array}$ & 147 \\
\hline
\end{tabular}

be overdiagnosed with $12.91 \%$ and $14.73 \%$, respectively (Table 5).

Table 6 provides summaries of the results of multivariate logistic regression. The number of cervical biopsy sites, age group, cytological results and transformation zone types appear as significant influences over colposcopic accuracy. Age of patients and HPV subtypes do not appear to be related $(p=0.504)$.

A greater the number of cervical biopsy sites appears to positively correlate with higher odds of accurate detection (OR 1.15, 95\% CI 1.05-1.25). Compared with NILM, patients whose cytologic results were ASC-US (OR 0.45, 95\% CI $0.325-0.613$ ) or LSIL (OR 0.48, 95\% CI 0.326$0.706)$ were significantly associated with decreased odds of detection accordance. Patients with TZ 2 (OR 0.55, 95\% CI 0.34-0.88) and TZ 3 (OR 0.52, 95\% CI 0.38-0.72) significantly correlated with decreased odds of detection accuracy, compared with TZ 1.

Table 7 presents the results of associations between patient characteristics and the accuracy of colposcopy in detecting HSIL+. We measured the detection of HSIL+ directly, according to an increasing number of lesion-directed biopsies, separately for women with one,
Table 6 Logistic regression analysis of factors influencing colposcopic accuracy in detecting HSIL+ $(n=1130)$

\begin{tabular}{lll}
\hline Variables & $\boldsymbol{\beta}$ & OR (95\% Cl) \\
\hline Cytology results & & 1.000 \\
NILM & -0.8060 & $0.447(0.325-0.613)$ \\
ASC-US & -0.7344 & $0.480(0.326-0.706)$ \\
LSIL & -1.6004 & $0.202(0.036-1.143)$ \\
ASC-H & -0.3302 & $0.719(0.250-2.067)$ \\
HSIL & & \\
HPV & & 1.000 \\
Negative & -0.1801 & $0.835(0.492-1.417)$ \\
Other HR-HPV positive & 0.2455 & $1.278(0.771-2.119)$ \\
HPV16/18 & & \\
Transformation zone type & & 1.000 \\
TZ 1 & -0.5993 & $0.549(0.342-0.883)$ \\
TZ2 & -0.6522 & $0.521(0.375-0.724)$ \\
TZ3 & 0.1355 & $1.145(1.052-1.246)$ \\
Number of biopsies & & \\
\hline
\end{tabular}

two and three or more biopsies. The accuracy of colposcopy significantly associated with numbers of biopsies among patients whose cytologic results $\leq$ NILM or those who were HPV negative or with other HR-HPV positive. Among all patients, the first biopsy increased from $52.87 \%$ in women with one directed biopsy to $79.44 \%$ in women with two lesion-directed biopsies $(p=0.027)$, reflecting the increasing severity of the cases. However, a third or more biopsies decreased the accuracy of cytology by $2.79 \%$ compared with two biopsies. By contrast, the accuracy for the first biopsy consistently decreased from $92.31 \%$ to $64.13 \%$ in women with three or more lesion-directed biopsies among patients with HPV negative or positive with other HR-HPV type $(p=0.004)$, and decreased from $89.74 \%$ to $79.80 \%$ among patients with cytology results $\leq$ NILM $(p=0.036)$.

\section{Discussion}

Cervical intraepithelial neoplasia and squamous intraepithelial lesions are considered pre-cancerous and often develop into invasive cervical carcinoma within 15 years of the original diagnosis. Unlike many conditions there is an opportunity for early diagnosis which improves outcomes, dramatically. However, neither screening nor 
Table 7 Associations between patient characteristics and the accuracy of colposcopy in detecting $\mathrm{HSIL}+$ with increasing number of lesion-directed biopsies $(n=1130)$

\begin{tabular}{|c|c|c|c|}
\hline Variables & Discordant & Concordant & $\mathbf{P}$ \\
\hline All $(n=1130)$ & & & 0.0265 \\
\hline \multicolumn{4}{|l|}{ Number of biospies } \\
\hline 1 & $47.13 \%(n=41)$ & $52.87 \%(n=46)$ & \\
\hline 2 & $20.56 \%(n=59)$ & $79.44 \%(n=228)$ & \\
\hline$\geq 3$ & $25.00 \%(n=63)$ & $75.00 \%(n=189)$ & \\
\hline Cytology $\leq$ NILM $(n=484)$ & & & 0.0362 \\
\hline \multicolumn{4}{|l|}{ Number of biospies } \\
\hline 1 & $10.26 \%(n=4)$ & $89.74 \%(n=35)$ & \\
\hline 2 & $17.41 \%(n=43)$ & $82.59 \%(n=204)$ & \\
\hline$\geq 3$ & $20.20 \%(n=40)$ & $79.80 \%(n=158)$ & \\
\hline $\begin{array}{l}\text { Cytology } \geq \text { ASC-US } \\
(n=101)\end{array}$ & & & 0.4779 \\
\hline \multicolumn{4}{|l|}{ Number of biospies } \\
\hline 1 & $14.29 \%(n=1)$ & $85.71 \%(n=6)$ & \\
\hline 2 & $40.00 \%(n=16)$ & $60.00 \%(n=24)$ & \\
\hline$\geq 3$ & $42.59 \%(n=23)$ & $57.41 \%(n=31)$ & \\
\hline $\begin{array}{l}\text { HPV-/Other HR- } \\
\text { HPV }+(n=190)\end{array}$ & & & 0.0038 \\
\hline \multicolumn{4}{|l|}{ Number of biospies } \\
\hline 1 & $7.69 \%(n=1)$ & $92.31 \%(n=12)$ & \\
\hline 2 & $17.65 \%(n=15)$ & $82.35 \%(n=70)$ & \\
\hline$\geq 3$ & $35.87 \%(n=33)$ & $64.13 \%(n=59)$ & \\
\hline HPV $16 / 18(n=395)$ & & & 0.4263 \\
\hline \multicolumn{4}{|l|}{ Number of biospies } \\
\hline 1 & $12.12 \%(n=4)$ & $87.88 \%(n=29)$ & \\
\hline 2 & $21.78 \%(n=44)$ & $78.22 \%(n=158)$ & \\
\hline$\geq 3$ & $18.75 \%(n=30)$ & $81.25 \%(n=130)$ & \\
\hline
\end{tabular}

later colposcopic examination are perfectly accurate for every individual, which means that women are often misdiagnosed or unnecessarily scared and embarrassed by unnecessary biopsies. Therefore, it is necessary to ensure we understand the factors which influence colposcopy. As such, an aim of this study was to assess agreement between colposcopy and cervical biopsy in identifying HSIL. Associated clinicopathological factors affecting diagnostic accuracy were then analyzed for research and development.

Records from 1130 patients admitted to SZMCHH from January up until $30^{\text {th }}$ December, 2018 were collected. Demographics and clinical characteristics including age, cytological examination results, HPV status, transformation zone type, number of cervical biopsy sites, colposcopy results and pathological results, were analyzed. The overall diagnostic accuracy of colposcopy in identifying HSIL+ was $69.64 \%$ which appears low. However, colposcopic accuracy has always been questioned because agreement between colposcopic diagnosis and cervical biopsy analysis varies between countries and even between hospitals. In this study, the sensitivity of colposcopic examination for detecting HSIL+ was $42.35 \%$, and the specificity was $77.60 \%$, which were similar to previous studies conducted in other cities of China $[6,14,15]$.

Agreement between colposcopic diagnosis and final pathology matched in $69.64 \%$ of cases in this study, which was also comparable to previous research in China. For example, $\mathrm{Li}$ et al. found agreement between colposcopic impression and histopathological diagnosis was $46.9 \%$ [15] using 2011 IFCPC colposcopic terminology, with a sensitivity for colposcopic examination at detecting HSIL+ at 54.7\%. Importantly, even though the Li et al. study was conducted in western China, there are differences which may not be based entirely upon sample size differences. As has been mentioned, colposcopic impressions are often biased by knowledge of a patient's history and previous tests administered. It was found by way of a systematic review that the positive predictive value for HSIL in the diagnosis of CIN2+ worldwide is 77.5\% [16]. Ouitrakul et al. reported that the accuracy of colposcopically directed biopsy to detect HSIL or more of the uterine cervix was $87.8 \%$ sensitive [17]. This shows that the diagnostic value of cytological HSIL results in the diagnosis of CIN2+lesions is reasonable yet this is not high enough to rely solely on cytology for cervical lesions.

These inaccuracies have prompted some to recommend a combination of tests, or serial co-testing, as this could improve accuracy; however, unnecessary testing impacts upon both the affordability of healthcare and individuals' psychological well-being. It should be noted that differences may also be due to the use of colposcopic terminology, as well as heterogeneous sample characteristics, and the level and experience of colposcopists. For example, in a large clinical study conducted in Australia with 18,421 satisfactory colposcopies performed between 1999 and 2004 , researchers found that colposcopy was $60 \%$ sensitive and with $60 \%$ PPV in identifying HSIL [18]. However, the PPVs in detecting HSIL [19] across included studies in a meta-analysis varied between 20 and 84\% [19]. This highlights issues which occur when synthesizing secondary retrospective data and does not provide reasons for such variability. Additionally, previous studies have emphasized specific, compulsory training for trainees before becoming qualified colposcopists, especially in low and middle-income countries [18].

We found a number of variables which are likely to influence colposcopic accuracy. In order to identify significant variables, we assessed colposcopic accuracy compared with final diagnosis in relation to different subgroups. We found that even though diagnostic accuracy of HSIL+ appeared to positively relate to increasing age 
in different patient groups, multivariate logistic regression analysis did not suggest age is an independent predictor factor. The reason for this might be heterogeneity within the sample or specific patient characteristics including TZ types and cytologic results. However, several clinical trials have reported that the risk of HSIL+actually decreases with increasing age among $\mathrm{HPV}+$ women under 40 years of age with negative cytology [20, 21]. This, the authors suggested, occurred because of age-related biological changes in the cervical transformation zone making the cervix less susceptible to new infection, or perhaps making small lesions more difficult to detect with a colposcope.

Some studies have also observed that the diagnostic accuracy of colposcopy-directed biopsy for identifying HSIL+ decreased as age increased. For example, Kim et al. [22] reported that the balanced accuracy of colposcopy-directed biopsy was $81 \%$ for those $<35$ years, $74.4 \%$ for $35-50$ years, and $68.8 \%$ for those older than 50 years. These findings highlight a decline in balanced accuracy with increasing age which Stuebs et al. [23] also found when studying accuracy rates for detecting HSILs using 2011 IFCPC colposcopic terminology, which were 93.1\% (age $0-34$ ), 83.6\% (age 34-55), and 80\% (age 55 or older). The authors themselves postulated that relatively poor diagnostic performances at identifying HSIL+cases in those $\geq 50$ years of age might be related to menopause and to unidentifiable squamocolumnar junctions or cervical lesions with limits that are not easily visualized using colposcopy although, this requires further research.

The number of cervical biopsy sites and cytologic results appeared in this study to be significantly related to colposcopic accuracy. We found an increasing number of cervical biopsy sites was significant, with 1.15 times higher odds of accurate detection. Gage et al. found that the sensitivity of enrollment colposcopic procedure did not vary significantly according to colposcopist's professional characteristics but that sensitivity was significantly greater when colposcopists took two or more biopsies [24], This finding has been confirmed by a study by Robert et al. [25] and Wentzensen et al. [26] who reported the highest sensitivity for detecting high-grade dysplasia was $95.6 \%$ after taking three biopsies according to the standards of the American Society for Colposcopy and Cervical Pathology (ASCCP). Although, it is not always the case that three of more biopsies will further improve identification. Zuchna et al. found that sensitivity increased after taking a second biopsy, but that there was no further improvement after taking a third biopsy [27].

Unlike previous research, we further found that the optimal number of biopsies depends on prior risk, as determined by cytology status, colposcopy impression, and HPV type status. Though the incremental benefit of taking two biopsies was present compared with only one site among all patients, our results suggested limited benefit from additional biopsies among patients with cytology results $\leq$ NILM or negative and other positive HPV types, which might even lead to the overdiagnosis of detecting HSIL+ cases using colposcopy. Our results were in accordance with previous findings [28]. Therefore, 'second-look' biopsies and perhaps even a third biopsy may be necessary to ensure we do not miss opportunities to intervene, and future study using larger sample sizes might be conducted to improve the performance of colposcopy based on stratified patient risks.

In this study, we found that $75.7 \%$ (NILM), 57.6\% (ASC-US), 60.4\% (LSIL) and 33.3\% (ASC-H) of cases with different cytology results had benign conclusions through colposcopic and pathologic examinations, with an accuracy rate of $73.7 \%$ in HSIL cases. The accuracy of colposcopy-directed biopsy was however lower for detecting LSIL when compared with HSIL, which was in line with previous studies. Tatiyachonwiphut et al. [29] found in colposcopic diagnosis of high-grade lesions, that pathologic determination will be either HSIL, MIC or invasive cancer in $75.5 \%$ of the study population. Conversely, in colposcopic diagnosis is low grade lesions, the cervical pathology will be normal, benign or LSIL in $83.8 \%$. A prospective multicenter trial in Austria reported that the sensitivity of colposcopically directed cervical biopsies was $66.2 \%$ [27]. Specifically, the agreement between histological results on biopsy and cone specimen was 54.5\% for low-grade lesions, $78.2 \%$ for high-grade lesions, and $28.9 \%$ for microinvasive cervical cancer [30]. Similarly, Howe and Vincenti [31] and Zuchna et al. [27] found that detection rates among LSIL women were around $31 \%$, while Baldauf et al. [32] reported an accuracy rate of over $80 \%$ for LSIL cases, which suggests there is a great degree of variability which demands further attention.

Loiudice et al. [33] found that agreement between LSIL and histologic results was $37 \%$, and agreement between HSIL and histologic results was $76 \%$ which appears incongruous with our findings. Our results suggest that $31.25 \%$ of all LSIL cases were underdiagnosed, yet only $8.33 \%$ were overdiagnosed. These findings were consistent across ASC-US and HSIL subgroups, and generally in line with the assertion that colposcopic impressions are more likely to overestimate rather than underestimate disease $[7,29]$. This maybe because various lesion points are inadvertently removed during biopsy, or may occur because practitioners had knowledge of the cytology results before colposcopy. Again, these not-sosubtle differences appear to relate to a colposcopists' skillsets or bias caused by assuming the biopsy is a form of colposcopic confirmation. The disagreement might be 
improved by advocating novel methods such as artificial intelligence used for grading colposcopic impressions and guiding biopsies [34, 35], as well as portable devices that can be used to perform colposcopy [36]. Both have shown potential in improving the diagnostic quality of colposcopy.

Among HPV-positive and HPV-negative patients, a higher percentage of overdiagnosis was observed, with the difference highly significant in HPV-negative patients. Zaal et al. [37] found a relatively high percentage of HPVwomen with high-grade lesions i.e. $22.8 \%$, which might be caused by false-negative HPV test results. Our study also found that among HPV + patients, the likelihood of underdiagnosis was higher, which was in line with previous studies [15]. However, the association between HPV subtypes and the accuracy of detecting HSIL+ using colposcopy was no longer significant under multivariate logistic regression. This suggests that the effect of HPV might diminish when assessed with other variables. Our study was not designed to determine whether different methods of HPV testing would bias colposcopic impressions although, it would seem prudent to investigate this further.

The 2011 IFCPC colposcopic terminology confirmed the classification of TZs as an obligatory terminology [6]. We found that the accuracy of detecting HSIL+in women decreased from TZ 1 to $\mathrm{TZ} 3$ (TZ 1, 79.8\%; TZ 2, 67.4\%; TZ 3, 65.7\%). This appears to be in accordance with previous findings $[23,30]$ which was to be expected due to the classifications themselves. The components of TZ 1 mean that the entire transformation zone is visible, whereas in TZ 3 the entire upper limit may not be visible and therefore can be misleading [23]. From a specialist's perspective, it seems necessary to focus further research on TZ 3 cases specifically because of the related diagnostic complexities. It appears the greatest number of false negative cases occur within this classification and therefore clinicians need additional indicators which might initiate a second-look biopsy or indeed a third where necessary. This is however, a fine balance between administering 'unnecessary testing' and managing patients' anxieties and well-being.

To the best of our knowledge, this is the first study to assess the diagnostic accuracy of colposcopy in identifying HSIL+among Chinese patients in Shenzhen, China. Populations in Chinese cities may be considered by some to be homogeneous but this is not the case and we also must look to understand potential differences between nationalities and ethnicities. Therefore, even though this study included only Chinese women it is necessary to develop our understanding of this population to make more reliable comparisons. This study was however retrospective with limited data availability which constrained our analysis. We tried to investigate the influence of colposcopists skills and experience although this was not included because we were unable to extract sufficient data to create subgroups. Of course, the relatively small sample sizes attributed to subgroups probably also affected our analysis of HPV subtypes, for example. The last major concern was that biopsy specimens were taken only from suspicious lesions without comparable control specimens.

\section{Conclusion}

The overall diagnostic accuracy of colposcopy and the consistency between colposcopy and histopathology in our study were comparable to previous studies, but there is room for improvement. The number of biopsies, cytology and transformation zone type appear to be predictors of misdiagnosis and therefore should be considered more carefully during clinical consultations and by way of further research. Future colposcopy-based studies using a reasonable scoring system and standard diagnostic criteria are still urgently needed to assess colposcopists' and colposcopic performance more objectively.

\section{Acknowledgements}

The authors would like to thank Shenzhen Maternity \& Child Healthcare Hospital for the support.

\section{Authors' contributions}

$A Y, J X$, and PX planned and designed the study. AY and JX performed the statistical analysis. AY, JX, and PX analyzed and interpreted the data. JX and QL collected the data. AY, PX and SS wrote the manuscript. AY, JX, QL, SS, PX and YJ provided conceptional assistance. All co-authors examined the manuscript, made corrections, and approved this publication. All authors read and approved the final manuscript.

\section{Funding}

This study was supported by CAMS Innovation Fund for Medical Sciences (CAMS 2021-12M-1-004).

\section{Availability of data and materials}

The datasets used and/or analyzed during the current study are available from the corresponding author on reasonable request.

\section{Declarations}

\section{Ethics approval and consent to participate}

This study was approved by the institutional review board of Shenzhen maternal and Child Health Hospital (No. 164). All methods were performed in accordance with the Declaration of Helsinki. The need for informed consent was waived by the IRB of SZMCHH due to the retrospective nature of archived datasets and fully anonymized personal information.

\section{Consent for publication}

All authors consent to the publication of this manuscript.

Competing interests

The authors declare that they have no conflicts of interest.

\section{Author details}

${ }^{1}$ Department of Epidemiology and Biostatistics, School of Population Medicine and Public Health, Chinese Academy of Medical Sciences and Peking Union Medical College, Beijing 100730, China. ${ }^{2}$ Diagnosis and Treatment for Cervical Lesions Center, Shenzhen Maternity \& Child Healthcare Hospital, 
Shenzhen 518028, China. ${ }^{3}$ Division of Health Research, Faculty of Health and Medicine, Lancaster University, Lancaster LA1 4YW, UK.

Received: 31 October 2021 Accepted: 31 December 2021 Published online: 11 January 2022

\section{References}

1. Sung H, Ferlay J, Siegel RL, Laversanne M, Soerjomataram I, Jemal A, et al. Global Cancer Statistics 2020: GLOBOCAN Estimates of Incidence and Mortality Worldwide for 36 Cancers in 185 Countries. CA Cancer J Clin. 2021;71(3):209-49.

2. Vu M, Yu J, Awolude OA, Chuang L. Cervical cancer worldwide. Curr Probl Cancer. 2018;42(5):457-65.

3. Chen W, Zheng R, Baade PD, Zhang S, Zeng H, Bray F, et al. Cancer statistics in China, 2015. CA Cancer J Clin. 2016;66(2):115-32.

4. Ziegert C, Wentzensen N, Vinokurova S, Kisseljov F, Einenkel J, Hoeckel $M$, et al. A comprehensive analysis of HPV integration loci in anogenital lesions combining transcript and genome-based amplification techniques. Oncogene. 2003;22(25):3977-84.

5. Sankaranarayanan R, Gaffikin L, Jacob M, Sellors J, Robles S. A critical assessment of screening methods for cervical neoplasia. Int J Gynaecol Obstet. 2005;89(Suppl 2):S4-s12.

6. Fan A, Wang C, Zhang L, Yan Y, Han C, Xue F. Diagnostic value of the 2011 International Federation for Cervical Pathology and Colposcopy Terminology in predicting cervical lesions. Oncotarget. 2018;9(10):9166-76.

7. Massad LS, Collins YC. Strength of correlations between colposcopic impression and biopsy histology. Gynecol Oncol. 2003;89(3):424-8.

8. Stafl A. New nomenclature for colposcopy. Report of the committee on terminology. Obstet Gynecol. 1976;48(1):123-4.

9. Stafl A, Wilbanks GD. An international terminology of colposcopy: report of the Nomenclature Committee of the International Federation of Cervical Pathology and Colposcopy. Obstet Gynecol. 1991;77(2):313-4.

10. Walker P, Dexeus S, De Palo G, Barrasso R, Campion M, Girardi F, et al. International terminology of colposcopy: an updated report from the International Federation for Cervical Pathology and Colposcopy. Obstet Gynecol. 2003;101(1):175-7.

11. Ruan Y, Liu M, Guo J, Zhao J, Niu S, Li F. Evaluation of the accuracy of colposcopy in detecting high-grade squamous intraepithelial lesion and cervical cancer. Arch Gynecol Obstet. 2020;302(6):1529-38.

12. Solomon D, Davey D, Kurman R, Moriarty A, O'Connor D, Prey M, et al. The 2001 Bethesda System: terminology for reporting results of cervical cytology. JAMA. 2002;287(16):2114-9.

13. Quaas J, Reich O, Frey Tirri B, Küppers V. Explanation and Use of the Colposcopy Terminology of the IFCPC (International Federation for Cervical Pathology and Colposcopy) Rio 2011. Geburtshilfe Frauenheilkd. 2013;73(9):904-7.

14. Li Y, Duan X, Sui L, Xu F, Xu S, Zhang H, et al. Closer to a uniform language in colposcopy: study on the potential application of 2011 international federation for cervical pathology and colposcopy terminology in clinical practice. Biomed Res Int. 2017;2017:8984516.

15. Li J, Wang W, Yang P, Chen J, Dai Q, Hua P, et al. Analysis of the agreement between colposcopic impression and histopathological diagnosis of cervical biopsy in a single tertiary center of Chengdu. Arch Gynecol Obstet. 2021;304(4):1033-41.

16. Karia N, Van Loon A, Simoens C, Benoy I, Bogers J. The positive predictive value of high-grade squamous intraepithelial lesion on cytology for the histological diagnosis of cervical intraepithelial neoplasia 2 or higher: a systematic review. Acta Cytol. 2019;63(3):206-14.

17. Ouitrakul S, Udomthavornsuk B, Chumworathayi B, Luanratanakorn S, Supoken A. Accuracy of colposcopically directed biopsy in diagnosis of cervical pathology at Srinagarind Hospital. Asian Pac J Cancer Prev APJCP. 2011;12(9):2451-3.

18. Bekkers RL, van de Nieuwenhof HP, Neesham DE, Hendriks JH, Tan J, Quinn MA. Does experience in colposcopy improve identification of high grade abnormalities? Eur J Obstet Gynecol Reprod Biol. 2008;141(1):75-8.

19. Mitchell MF, Schottenfeld D, Tortolero-Luna G, Cantor SB, Richards-Kortum R. Colposcopy for the diagnosis of squamous intraepithelial lesions: a meta-analysis. Obstet Gynecol. 1998;91 (4):626-31.
20. Castle PE, Jeronimo J, Schiffman M, Herrero R, Rodríguez AC, Bratti MC, et al. Age-related changes of the cervix influence human papillomavirus type distribution. Can Res. 2006;66(2):1218-24.

21. Critchlow CW, Wölner-Hanssen P, Eschenbach DA, Kiviat NB, Koutsky LA, Stevens CE, et al. Determinants of cervical ectopia and of cervicitis: age, oral contraception, specific cervical infection, smoking, and douching. Am J Obstet Gynecol. 1995;173(2):534-43.

22. Kim SI, Kim SJ, Suh DH, Kim K, No JH, Kim YB. Pathologic discrepancies between colposcopy-directed biopsy and loop electrosurgical excision procedure of the uterine cervix in women with cytologic high-grade squamous intraepithelial lesions. J Gynecol Oncol. 2020;31(2):e13.

23. Stuebs FA, Schulmeyer CE, Mehlhorn G, Gass P, Kehl S, Renner SK, et al. Accuracy of colposcopy-directed biopsy in detecting early cervical neoplasia: a retrospective study. Arch Gynecol Obstet. 2019;299(2):525-32.

24. Gage JC, Hanson VW, Abbey K, Dippery S, Gardner S, Kubota J, et al. Number of cervical biopsies and sensitivity of colposcopy. Obstet Gynecol. 2006;108(2):264-72.

25. Pretorius RG, Belinson JL, Burchette RJ, Hu S, Zhang X, Qiao YL. Regardless of skill, performing more biopsies increases the sensitivity of colposcopy. J Low Genit Tract Dis. 2011;15(3):180-8.

26. Wentzensen N, Walker JL, Gold MA, Smith KM, Zuna RE, Mathews C, et al. Multiple biopsies and detection of cervical cancer precursors at colposcopy. J Clin Oncol. 2015;33(1):83-9.

27. Zuchna C, Hager M, Tringler B, Georgoulopoulos A, Ciresa-Koenig A, Volgger $B$, et al. Diagnostic accuracy of guided cervical biopsies: a prospective multicenter study comparing the histopathology of simultaneous biopsy and cone specimen. Am J Obstet Gynecol. 2010;203(4):321.e1-6.

28. Wentzensen N, Schiffman M, Silver MI, Khan MJ, Perkins RB, Smith KM, et al. ASCCP colposcopy standards: risk-based colposcopy practice. J Low Genit Tract Dis. 2017;21 (4):230-4.

29. Tatiyachonwiphut M, Jaishuen A, Sangkarat S, Laiwejpithaya S, Wongtiraporn W, Inthasorn P, et al. Agreement between colposcopic diagnosis and cervical pathology: Siriraj hospital experience. Asian Pac J Cancer Prev APJCP. 2014;15(1):423-6.

30. Fan A, Zhang L, Wang C, Wang Y, Han C, Xue F. Analysis of clinical factors correlated with the accuracy of colposcopically directed biopsy. Arch Gynecol Obstet. 2017;296(5):965-72.

31. Howe DT, Vincenti AC. Is large loop excision of the transformation zone (LLETZ) more accurate than colposcopically directed punch biopsy in the diagnosis of cervical intraepithelial neoplasia? Br J Obstet Gynaecol. 1991;98(6):588-91.

32. Baldauf JJ, Dreyfus M, Ritter J, Philippe E. An analysis of the factors involved in the diagnostic accuracy of colposcopically directed biopsy. Acta Obstet Gynecol Scand. 1997;76(5):468-73.

33. Loiudice L, Abbiati R, Boselli F, Cecchini G, Costa S, Grossi E, et al. Improvement of Pap smear sensitivity using a visual adjunctive procedure: a co-operative Italian study on speculoscopy (GISPE). Eur J Cancer Prev. 1998;7(4):295-304.

34. Xue P, Tang C, Li Q, Li Y, Shen Y, Zhao Y, et al. Development and validation of an artificial intelligence system for grading colposcopic impressions and guiding biopsies. BMC Med. 2020;18(1):406.

35. Xue P, Ng MTA, Qiao Y. The challenges of colposcopy for cervical cancer screening in LMICs and solutions by artificial intelligence. BMC Med. 2020;18(1):169.

36. Taghavi K, Rohner E, Basu P, Low N, Rutjes A, Bohlius J. Screening test accuracy of portable devices that can be used to perform colposcopy for detecting CIN2+ in low- and middle-income countries: a systematic review and meta-analysis. BMC Womens Health. 2020;20(1):253.

37. Zaal A, Louwers JA, Berkhof J, Kocken M, Ter Harmsel WA, Graziosi GC, et al. Agreement between colposcopic impression and histological diagnosis among human papillomavirus type 16-positive women: a clinical trial using dynamic spectral imaging colposcopy. BJOG Int J Obstet Gynaecol. 2012;119(5):537-44.

\section{Publisher's Note}

Springer Nature remains neutral with regard to jurisdictional claims in published maps and institutional affiliations. 